\title{
Searching for Optical Visual Double Stars
}

\author{
D. SINACHOPOULOS ${ }^{1} \&$ P. MOUZOURAKIS \\ ${ }^{1}$ Royal Belgian Observatory, Ringlaan 3, B-1180 Brussels, Belgium
}

\begin{abstract}
We study a sample of 123 wide visual double stars for which astrophysical parameters are at least partly available from $u v b y H \beta$ photometry. All of these have components brighter than 10 mags and a component angular separation between 10 and 20 arcseconds.

We find that at least one third of them are optical; for relative proper motion (RPM) pairs this fraction is $60 \%$ or even more. These results are in fair agreement with the predictions we formulated in an earlier paper on the basis of a statistical criterion.
\end{abstract}

\section{INTRODUCTION}

The interest devoted to visual double stars and the various attempts to date to formulate criteria that would permit a reliable distinction between physical and optical pairs to be made, can be easily explained with the wealth of information on stellar structure and evolution which can be obtained from the study of visual binaries. Heintz (1978) and Halbwachs (1988) comprehensively review such criteria.

It is however apparent from the two recent investigations of Brosche et al. (1992) and of Sinachopoulos \& Mouzourakis (1991a) that visual double star catalogues still contain a considerable number of optical pairs, no doubt due to the poor accuracy of astrometric information as well as the paucity of astrophysical data.

On the basis of a purely statistical argument, the authors of the second study concluded that there are at least $3000(13.5 \%)$ physical pairs in Aitken's Double Star Catalogue (1932) (Sinachopoulos \& Mouzourakis 1991b) and at least $6500(8.7 \%)$ in the Washington Double Star Catalogue (WDS, Worley \& Douglass 1984) (Sinachopoulos \& Mouzourakis 1991c).

\section{OBSERVATIONAL DATA ANALYSIS}

We have considered a sample of wide visual double stars such that the component separation was between 10 and 20 arcseconds, while both the primary and the secondary were brighter than 10 mags.

They are contained in four different published studies, each with emphasis on a particular type of wide visual pairs: Lindroos(1985) has studied early type primary ( $\mathrm{A} 0$ and earlier) pairs, mostly with at least two magnitudes difference of their components. Sinachopoulos (1991), on the other hand, studied F or G0 type primaries and small brightness differences. Two other publications (Sinachopoulos $1989 \& 1990$ ) studied subsamples of the astrometric HIPPARCOS Input Catalogue having either significant relative proper motion (RPM) of the two components or common proper motion (CPM) without restriction on primary types or brightness difference. While we make no claim that the total sample compiled from these four publications is representative of the double 
star population as a whole, it does not seem to specifically exclude any subset of wide visual double stars. The total number of pairs thus obtained was 123 or $11.4 \%$ of the total number of entries in the WDS satisfying our cuts; for primary and secondary both brighter than 9 mags the corresponding percentage is even higher: $17.3 \%$.

We tried to avoid the use of mild criteria for the opticity of visual pairs, limiting ourselves to only two tests: agreement of the distances obtained by $u v b y H \beta$ photometry for the two components of a given pair and compatibility of the observed relative proper motion with that dynamically allowed.

Thus, for 15 of the 123 pairs no reliable distance can be assigned to either component. For 2 of these 15 however, Lindroos shows that the two components cannot be compatible with a common distance and are hence optical.

Of the 108 remaining, 22 have distance of the secondary which differs by more than $2 \sigma$ (primary) from that of the primary: these are also optical.

Of the remaining 86 , for some of which only one component distance is available, 56 are CPM pairs and 30 RPM pairs, to which now a further test can be applied: consider $V_{\text {tan }}$ (in kms per sec), the tangential velocity corresponding to their observed relative proper motion $\mu$ in arcsecs per century:

$$
V_{\text {tan }}=\frac{4.74}{10^{2}} \cdot d \cdot \mu
$$

where $d$ is in parsecs.

If the pair under consideration is indeed bound, $V_{\tan }$ should be less than or equal to the maximum orbital velocity that follows from Kepler's third law:

$$
V_{\text {orb }}=29.78 \cdot \sqrt{\frac{\mathcal{M}}{s}}
$$

again in kms per sec, where $s$, in astronomical units is the projected separation (distance times angular separation), which is less than or equal to the radius of the (assumed circular) orbit and $\mathcal{M}$ is the total component mass expressed in solar units. Our criterion for an RPM pair to be optical is then: $V_{\tan }-2 \cdot \sigma>$ $V_{\max }$ where $\sigma$ is the uncertainty in $V_{\tan }$ due to the measured error on $\mu$. We have calculated $V_{\max }$ using the values given by Schmidt-Kaler (1982) for mass as a function of the primary and secondary spectral type and luminosity class. In practice, for all cases where a pair turns out to be optical $V_{\text {tan }}$ is at least 3 times as great as $V_{\max }$, sometimes more than an order of magnitude, while many of the surviving pairs are due to large errors in $\mu$. Anyway, 18 pairs out of the 30 RPM ones are optical on the basis of the above criterion.

It is interesting to point out that all "non-optical" RPM pairs have $s<2000$ A.U. In fact, all 14 RPM pairs with $s>2000$ are optical, which yields an upper limit of $2.3 / 14=16.5 \%$ (2.3 is the Poisson parameter corresponding to $90 \%$ probability) for the fraction of physical RPM pairs with $s>2000$ A.U. in the magnitude and separation region considered.

For the CPM pairs no obvious equivalent of the above test exists. By considering that all CPM pairs are physical, we have found a lower limit of at least $2+22+18=42$ clearly optical pairs out of a total of 123 , i.e. more than a third. For primary and secondary mags $<9$ we find 8 surely optical pairs out of 47 , i.e. about a sixth. 


\section{DISCUSSION}

We can compare the number of optical pairs found in the preceding section to the upper limit for the total number of optical pairs in the sky predicted by our statistical model:

$$
N_{\text {opt }} \simeq \frac{2.424}{10^{7}} \cdot N_{m_{1}} \cdot D_{m_{2}} \cdot \rho^{2}
$$

where $N_{m_{1}}$ is the total number of stars with magnitude $m_{1}$ in the sky, and $D_{m_{2}}$ is the density of magnitude $m_{2}$ stars given in stars per square degree.

Using the Scheffler-Elsässer (1965) tables for stellar densities, renormalized so as to yield the correct number of bright stars $(\mathrm{mag}<6)$ found in the Bright Star Catalogue (Hoffleit \& Jaschek 1982) we obtain 22 such pairs in the range of $\rho^{\prime \prime}$ and magnitudes considered.

Our analysis of observational data indicates at least twice as many. Given the uncertainties inherent in the data, as well as the possibility of bias in our sample, however, the agreement can be considered as fair. We are confident thus that the statistical model can provide at least a lower bound to the fraction of optical pairs present in double star catalogues.

\section{ACKNOWLEDGMENTS}

D. Sinachopoulos gratefully acknowledges financial support from the European Community. We would also like to express our thanks to J. L. Halbwachs for useful remarks.

\section{REFERENCES}

Aitken R.G. 1932, New General Catalogue of Double Stars within $120^{\circ}$ of the North Pole (Carnegie Inst. Washington Publ.)

Brosche et al. 1992, $A \& A, 253,113$

Halbwachs J.L. 1988, Ap\&SS, 142, 237

Heintz W.D. 1978, Double Stars, Geophysics and Astrophysics Monographs, (Dordrech, Reidel), vol. 15

Hoffleit, D. \& Jaschek, C. 1982, The Bright Star Catalogue, 4th ed., (New Haven, Yale University)

Lindroos K. P. 1985, $A \mathscr{E} A S, 60,183$

Scheffler H., Elsässer H. 1965, in Landolt-Börnstein, Vol I, $A \& A$

Schmidt-Kaler Th. 1982, in Landolt-Börnstein, New Series, VI/2b, (Heidelberg, Springer), p. 604

Sinachopoulos D. 1989, $A \mathscr{S A S}, \mathbf{8 1}, 103$

Sinachopoulos D. $1990, A \& A S, 86,75$

Sinachopoulos D. 1991, $A \mathcal{E A S}, 87,453$

Sinachopoulos D. \& Mouzourakis P. 1991a, $A \& A, 245,513$

Sinachopoulos D. \& Mouzourakis P. 1991b, Astron. Ges. Abstr. Ser., 6, 84

Sinachopoulos D. \& Mouzourakis P. 1991c, Bull. Inst. Math. Statistics, 20, No. 3, 274 Worley C.E. \& Douglass G.G. 1984, The Washington Visual Double Star Catalog, (Washington, U.S. Naval Obs.) 